\section{Aktinische Keratosen: PDT auch mit Weißlicht}

\author{
Bei einer photodynamischen Therapie bei aktinischer Keratose muss der \\ Patient weder Schmerzen in Kauf nehmen noch sich stundenlang im Freien \\ aufhalten. Wie eine Studie gezeigt hat, erzielt man auch mit einer \\ gewöhnlichen Weißlichtquelle gute Effekte.
}

$D^{2}$ ie photodynamische Therapie mit Tageslicht (DPDT, „daylight photodynamic therapy") ist eine beliebte Methode zur Behandlung aktinischer Keratosen (AK). Bei dieser Variante wird wie bei der konventionellen PDT der Lichtsensibilisator (5-Aminolävulinsäure, ALA, oder Methyl-5-Amino-4-Oxopentanoat, MAOP) auf die betroffenen Stellen aufgetragen. Die Wartezeit bis zur Lichtexposition beträgt jedoch nur eine halbe Stunde.

Die Tageslichtmethode ist zudem mit deutlich weniger Schmerzen verbunden als die herkömmliche PDT mit kaltem Rotlicht. Dies hängt damit zusammen, dass die aktive Substanz, Protoporphyrin IX (PpIX), unter der zweistündigen Tageslichtexposition kontinuierlich gebildet und in dem Maße wieder inaktiviert wird, wie sie aus der Vorläufersubstanz entsteht; so werden Entzündungsreaktionen zu einem Großteil vermieden. Einer der Nachteile der DPDT ist jedoch der erzwungene mehrstündige Aufenthalt im Freien. Eine mögliche Lösung ist die PDT mit einer künstlichen weißen Lichtquelle (AWLPDT, ,artificial white light PDT“).

Irische Dermatologen haben für ihre Studie 22 Männer (Durchschnittsalter 72) mit weitgehend gelichtetem Haupthaar herausgesucht. Die Kopfhaut der Teilnehmer zeigte deutliche lichtassoziierte Hautschäden und eine Vielzahl von AK. Bei allen Patienten kamen nacheinander DPDT und AWLPDT zum Einsatz, und zwar auf verschiedenen, voneinander abgegrenzten Kopfhaut- und Stirnarealen, wobei jeweils nur ein Areal mit einem MAOP-Präparat eingecremt wurde. Zwischen Tageslicht- und Weißlichtbehandlung lag im Mittel eine Woche. Als Lichtquelle für Letztere diente eine OP-Lampe. Die Dauer der Exposition betrug jeweils zwei Stunden.

Die mit Tageslicht erreichte effektive Dosis lag bezogen auf das Absorptionsspektrum von PpIX im Mittel bei 21,38 $\mathrm{J} / \mathrm{cm}^{2}$. Auch im Hautfeld, das das Licht der OP-Lampe abbekommen hatte, waren die effektiven Dosen unterschiedlich, sie nahmen vom Zentrum zu den Rän-

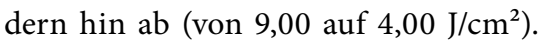
Nach den Sitzungen wurden die Patienten neun Monate lang nachbeobachtet. Auf jeweils beiden Arealen waren die Stellen mit AK bereits nach einem Monat deutlich zurückgegangen, im Mittel von 20,5 auf 8,5 im DPDT-Feld und auf 7,5 im AWLPDT-Feld. Die Reduktion der aktinischen Keratosen betrug nach einem Monat $62,3 \%$ und 67,7\%.

Die Unterschiede waren zu keinem Zeitpunkt signifikant. Der Effekt hielt auf beiden Seiten über die gesamte Beobachtungszeit an, wenngleich sich teilweise neue AK gebildet hatten. Schmerzen waren gemessen anhand einer visuellen Analagskala in beiden Gruppen etwa gleich niedrig.

Fazit: Die Weißlicht-PDT erwies sich als ebenso effektiv und wenig schmerzhaft wie die PDT mit Tageslicht. Der Vorteil: Die Patienten können im Inneren bleiben. Die Therapie kann zudem ganzjährig durchgeführt werden. Die Forscher empfehlen eine etwa einmal jährliche Wiederholung der Therapie zur Behandlung etwaiger Rezidive.

Dr. Elke Oberhofer

O'Gorman SM et al. Artificial White Light vs Daylight Photodynamic Therapy for Actinic Keratoses: A Randomized Clinical Trial. JAMA Dermatol 2016; 152: 638-44

\section{Die Krätze arbeitet sich durch die Haut}

Eine 61-jährige Frau berichtete über einen seit sechs Monaten bestehenden starken Juckreiz. An Armen, Beinen, Bauch und Rücken fanden sich multiple aufgekratzte Papeln mit honigfarben Krusten (Abb. A). Unter der Verdachtsdiagnose einer Follikulitis und einer Prurigo nodularis wurde über vier Wochen eine Therapie mit Fluocinonid-Creme und Doxycyclin durchgeführt, die aber nichts an den Effloreszenzen änderte. Bioptisch zeigte sich ein eosinophiles Infiltrat, das an ein Arzneimittelexanthem erinnerte. Daraufhin wurden sämtliche Medikamente abgesetzt, was auch zu keiner Besserung führte.

Die Diagnose konnte schließlich mithilfe eines Schabepräparats von den Händen gestellt werden. Unter dem Mikroskop erkannte man eine Scabiesmilbe (Abb. B). Mit PermethrinCreme behandelt bildeten sich die Effloreszenzen innerhalb von zwei Wochen zurück. Typisch für die Scabies ist ein starker Juckreiz, der bei der Bettwärme nachts zunimmt. Ohne Behandlung drohen eine sekundäre Dermatitis aufgrund des Kratzens sowie Impetigo, Ekthyma, Paronychien oder Furunkulose. Die an sich mit bloßem Auge erkennbaren Milbengänge in der Haut können durch Kratzeffloreszenzen und Impetiginisierung maskiert werden. Biopsien zeigen oft unspezifische Veränderungen ohne Milbengänge und Milben, da manchmal nur 6-10 Milben im gesamten Integument vorhanden sind. Insofern ist der hier gezeigte Befund als Glückstreffer zu bezeichnen. Mit der gegenwärtigen Migrationswelle wird uns die Scabies wohl wieder mehr beschäftigen. Prof. Hermann S. FüeßI

Carr PC et al. Scabies. N Engl J Med 2016; 374: e13
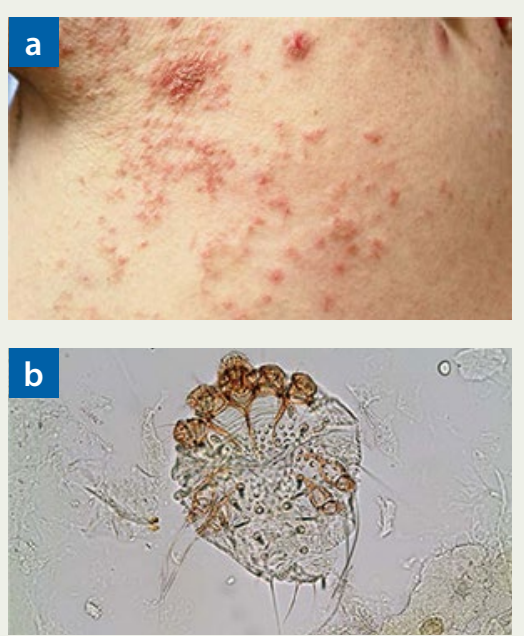

Multiple juckende, aufgekratzte $\mathrm{Pa}$ peln mit honigfarben Krusten (A); Scabiesmilbe in der mikroskopischen Darstellung (B). 